\section{Fatores associados ao excesso de peso entre adolescentes de diferentes redes de ensino do município de Presidente Prudente, São Paulo}

\section{Factors associated with overweight among adolescents in different school systems in the municipality of Presidente Prudente in the State of São Paulo}

Rômulo A. Fernandes 1 Jamile S. Codogno 2 Jefferson R. Cardoso 3 Enio R. V. Ronque 4 Ismael F. Freitas Júnior 5 Arli R. de Oliveira 6

\section{Abstract}

Objective: to analyze associations between overweight and family risk factors in different school environments.

Methods: a descriptive/analytical cross-sectional study of 1779 adolescents (aged between 11 and 17 years) of both sexes in the public $(n=1309)$ and private $(n=470)$ education systems. Family factors evaluated included: sex, economic circumstances, number of siblings, number of televisions and level of schooling of parents. The prevalence ratio $(P R)$ in a univariate and multivariate model suggested the scale of the associations under study.

Results: in private schools, overweight was associated with the level of schooling of the father $(R P=$ 0.68 [0.5-0.9]) and with boys ( $R P=1.38$ [1.0-1.8]). In public schools it was associated with the level of schooling of the mother ( $R P=1.42$ [1.1-1.8]), having fewer siblings $(R P=1.23[0.9-1.5])$ and with boys $(R P=1.32[1.6-1.6])$.

Conclusions: overweight is associated with different family factors depending on the kind of school.

Key words Overweight, Risk factors, Motor activity, Food intake

\section{Resumo}

Objetivo: analisar associações entre excesso de peso e fatores de risco familiares em diferentes âmbitos escolares.

Métodos: estudo descritivo/analítico de delineamento transversal com 1779 adolescentes (11 a 17 anos) de ambos os sexos, da rede pública $(n=1309) e$ privada de ensino $(n=470)$. Foram avaliados os fatores familiares: sexo, condição econômica, número de irmãos/televisores e escolaridade dos pais. A razão de prevalência $(R P)$ em modelo univariado e multivariado indicou a magnitude das associações analisadas.

Resultados: nas escolas particulares, o excesso de peso associou-se com a escolaridade paterna ( $R P=$ $0,68[0,5-0,9])$ e sexo masculino $(R P=1,38[1,0$ 1,8]). Nas escolas públicas, com a escolaridade materna $(R P=1,42[1,1-1,8])$, menor número de irmãos $(R P=1,23[0,9-1,5])$ e sexo masculino $(R P=$ $1,32[1,6-1,6])$.

Conclusões: o excesso de peso associou-se com diferentes fatores familiares, dependendo da rede de ensino em que foi analisado.

Palavras-chave Sobrepeso, Fatores de risco, Atividade motora, Ingestão alimentar 
Introdução

Estudos recentes indicam que a ocorrência do excesso de peso aumentou significativamente nas últimas décadas entre a população brasileira, ${ }^{1}$ especialmente entre crianças e adolescentes. ${ }^{2} \mathrm{O}$ aumento expressivo na ocorrência desse fenômeno constitui um importante motivo de preocupação, uma vez que o excesso de adiposidade corporal, mesmo durante a infância e a adolescência, está associado ao surgimento de doenças crônicas não transmissíveis, como a hipertensão arterial, resistência à insulina e perfil lipídico desfavorável.3,4

Ingestão calórica elevada e diminuição da atividade física têm sido apontadas como os principais fatores responsáveis pelo aumento na ocorrência da obesidade. Evidências sólidas têm indicado que fatores comportamentais e núcleo familiar associamse com expressiva magnitude ao processo de desenvolvimento do excesso de peso.5,6 No que se refere às informações provenientes da população brasileira, a condição socioeconômica também é um fator determinante no surgimento da obesidade, 2 uma vez que um nível socioeconômico elevado propicia maior acesso a alimentos e bens de consumo, interferindo também no nível de atividade física. 7

Pesquisas em diferentes regiões do país apontam maior ocorrência de excesso de peso/perfil lipídico desfavorável entre crianças e adolescentes matriculados em unidades escolares da rede privada de ensino ${ }^{7-12}$ e, consequentemente, atribuem tal fenômeno à associação positiva existente entre excesso de peso e renda familiar.5,7,8 Entretanto, existem outras diferenças significativas entre os comportamentos de adolescentes da rede privada e pública, ${ }^{7}$ apontando assim, uma significativa contribuição do meio escolar no combate/promoção do desenvolvimento do sobrepeso e obesidade $(\mathrm{S} / \mathrm{O})$.

Até a presente data, porém, nenhum estudo nacional se propôs a analisar de maneira distinta as associações entre diferentes fatores de risco e a presença do $\mathrm{S} / \mathrm{O}$ entre adolescentes das redes privada e pública de ensino. Tal discernimento sobre o impacto de diferentes âmbitos escolares no desenvolvimento do excesso de peso poderá contribuir para a estruturação de políticas públicas mais eficientes de combate ao mesmo, uma vez que o meio escolar pode constituir um importante agente de ação sobre fatores de risco à saúde entre crianças e adolescentes. 13

Sendo assim, o objetivo do presente estudo foi analisar, de maneira separada, a associação entre diferentes fatores familiares e a presença do S/O entre adolescentes das redes privada e pública de ensino.

\section{Métodos}

Pesquisa de delineamento transversal, com características descritivas/analíticas conduzida durante o segundo semestre do ano de 2007 na cidade de Presidente Prudente $(200.000$ habitantes e IDH $=$ 0,846), localizada na Região oeste do Estado de São Paulo e que abriga aproximadamente 36.000 alunos (23.500 - 11 a 17 anos) em 118 unidades escolares particulares e públicas $(\sim 30 \%$ dos alunos matriculados na rede privada). Na referida cidade, entre as 118 unidades escolares, apenas 36 (24 públicas e 12 privadas) atendiam adolescentes de 11 a 17 anos. Assim, respeitando a distribuição dos alunos nas redes, entre essas 36 instituições de ensino, seis (quatro públicas e duas privadas) foram selecionadas aleatoriamente para participar do estudo.

Para a realização do estudo, foram efetuados três cálculos amostrais mínimos: a) escolas públicas ( $\mathrm{n}=$ 657 [prevalência de 28.6; erro de 3,5\%; poder de $80 \%$; intervalo de confiança de $95 \%]$ ); b) escolas particulares $(\mathrm{n}=310$ [prevalência de 28.6; erro de $4,5 \%$; poder de $80 \%$; intervalo de confiança de $95 \%])$; e c) amostra total $(\mathrm{n}=1779)$. O tamanho da amostra de 1779 alunos foi calculado para detectar uma prevalência esperada de S/O igual a $28,6 \%, 14$ com um erro amostral de $2,1 \%$, poder de $80 \%$ e significância estatística de 5\%. Prevendo perdas de aproximadamente $12,5 \%$, planejou-se coletar informações referentes a no mínimo 2000 adolescentes de ambos os sexos. Após a exclusão dos indivíduos que apresentaram alguma irregularidade nos dados fornecidos/coletados, a amostra final foi composta por 1779 adolescentes de 11 a 17 anos, na qual todos os cálculos de tamanho amostral foram alcançados e respeitou-se a proporção entre as redes (públicas: $n=1309$ /particulares: $n=470$ [26,4\%]).

Os critérios para a inclusão dos jovens na amostra do estudo foram baseados em três informações: a) Estar devidamente matriculado em uma das seis instituições de ensino em questão; b) Não apresentar nenhum tipo de doença metabólica diagnosticada, necessidade especial que interferisse no resultado e estar em processo de gestação; c) Retornar com o Termo de Consentimento Livre e Esclarecido devidamente assinado. O estudo foi aprovado pelo Comitê de Ética em Pesquisa envolvendo Seres Humanos da Universidade Estadual Paulista - UNESP (Presidente Prudente).

O preenchimento de todos os questionários e as medidas antropométricas foram realizados em uma sala reservada dentro da própria unidade escolar. A idade cronológica foi determinada em forma centesimal, utilizando as datas de nascimento e o dia da 
avaliação. Seguindo metodologia conhecida, 15 a massa corporal (balança mecânica da marca Filizola - precisão: 0,1 kg; capacidade máxima: $150 \mathrm{~kg}$ ) e a estatura (estadiômetro fixo de madeira - precisão: $0,1 \mathrm{~cm}$; extensão máxima: dois metros) foram aferidas para o cálculo do índice de massa corporal (IMC). A caracterização do excesso de peso foi feita por meio de valores de IMC. Para tanto, foram utilizados os pontos de corte ajustados por idade e sexo, previamente propostos por Cole et al.16 (Tabela 1), através dos quais cada sujeito da amostra foi classificado segundo o respectivo estado nutricional (peso normal, sobrepeso ou obesidade). Em todas as análises do estudo, os jovens com sobrepeso e obesidade foram unidos em um único grupo que, por sua vez, caracterizou o excesso de peso.

Hábitos alimentares e atividade física foram tratados como possíveis variáveis de confusão e utilizadas no modelo ajustado da regressão de Poisson. O preenchimento dos questionários foi realizado com o auxílio de um único avaliador, que efetuou breve explicação sobre a forma correta de preenchimento e respondeu questionamentos dos alunos. Não houve comunicação entre os avaliados durante o preenchimento.

As informações referentes à prática habitual de atividades físicas foram levantadas com a utilização do questionário desenvolvido por Baecke et al.17 Foram considerados suficientemente ativos os jovens que declararam estar engajados em alguma atividade esportiva de intensidade moderada/vigorosa, por um período mínimo de três meses antes da avaliação e que despendesse nessa tarefa mais do que $4 \mathrm{~h} /$ semana.

Os hábitos alimentares foram coletados por meio de um instrumento desenvolvido especificamente para o estudo. Ele foi composto por quatro perguntas, onde os avaliados responderam com que frequência semanal (nenhum dia; 1 a 2 dias; 3 a 5 dias; todos os dias da semana) consumiram determinados tipos de alimentos (legumes e verduras; doces, salgados e refrigerantes; frutas; frituras) na semana anterior à realização da avaliação. Para "legumes e verduras" e "frutas" foi considerado fator de risco para o $\mathrm{S} / \mathrm{O}$ a resposta "nenhum dia", já para "doces, salgados e refrigerantes" e "frituras" foi considerada a resposta "todos os dias da semana". Os adolescentes que apresentaram simultaneamente os quatro fatores de risco foram inseridos no grupo "alimentação inadequada". Dados sobre a reprodutibilidade desse instrumento (intervalo de uma semana entre as duas medidas) foram coletados em 170 adolescentes (selecionados randomicamente) e indicados pelo índice Kappa (k), que indicou alta concordância (Frutas $[\mathrm{k}=0,75]$; Frituras $[\mathrm{k}=0,76]$; Legumes e verduras $[\mathrm{k}=0,75]$; doces salgados e refrigerantes $[\mathrm{k}=0,76]$; mesma resposta nos quatro itens $[k=$ $0,87])$.

Os fatores familiares analisados no presente estudo foram: sexo, condição econômica (CE), número de aparelhos televisores na residência, escolaridade paterna/materna e número de irmãos. $\mathrm{Na}$ determinação da condição econômica foram empregados os "Critérios de Classificação Econômica do Brasil” (ANEP), 18 que estabelece uma classificação de A (mais alta) até E (mais baixa) para CE. Assim, a amostra foi subdividida em CE alta (CEA: categorias A e B) e baixa (CEB: categorias C, D e E). Deste questionário foram retiradas também informações referentes à quantidade de aparelhos televisores na residência. Os pais foram convidados a preencher em casa um questionário com informações referentes à respectiva escolaridade e, também, número de irmãos morando na mesma residência do adolescente em questão.

Tabela 1

Valores críticos internacionais para índice de massa corporal utilizados na indicação do excesso de peso.

\begin{tabular}{lcccc}
\hline & \multicolumn{2}{c}{ Masculino $\mathbf{( k g / \mathbf { m } ^ { 2 } )}$} & \multicolumn{2}{c}{ Feminino $\mathbf{( k g / \mathbf { m } ^ { 2 } )}$} \\
\cline { 2 - 5 } $\begin{array}{l}\text { Grupos etários } \\
\text { (em anos) }\end{array}$ & Sobrepeso & Obesidade & Sobrepeso & Obesidade \\
\hline 11,5 & 20,89 & 25,58 & 21,20 & 26,05 \\
12,5 & 21,56 & 26,43 & 22,14 & 27,24 \\
13,5 & 22,27 & 27,25 & 22,98 & 28,20 \\
14,5 & 22,96 & 27,98 & 23,66 & 28,87 \\
15,5 & 23,60 & 28,60 & 24,17 & 29,29 \\
16,5 & 24,19 & 29,14 & 24,54 & 29,56 \\
17,5 & 24,73 & 29,70 & 24,85 & 29,84 \\
\hline
\end{tabular}

Adaptado de Cole et al. ${ }^{16}$ 
Com o objetivo de se avaliar a consistência das informações fornecidas por todos os questionários, duas semanas após a coleta dos dados uma pequena parcela da amostra (170 alunos e 30 pais), selecionada de forma aleatória, preencheu novamente os questionários, sendo analisado o grau de concordância (índice Kappa) das respostas (atividade física $[\mathrm{k}=0,85], \mathrm{CE}[\mathrm{k}=0,87]$ e dados dos pais $[\mathrm{k}=1,00])$.

$\mathrm{O}$ teste de Kolmogorov-Smirnov indicou a necessidade de se utilizar estatística não-paramétrica no tratamento das variáveis numéricas (idade, massa corporal, estatura e IMC) e o teste U de MannWhitney estabeleceu comparação entre as mesmas. $\mathrm{O} k$ indicou a concordância dos dados categóricos. $\mathrm{O}$ teste qui-quadrado $\left(\chi^{2}\right)$ analisou a associação entre as variáveis e também comparou proporções. A regressão de Poisson com ajuste robusto de variância, representada por valores de razão de prevalência (RP) e intervalo de confiança de 95\% (IC95\%) indicou a magnitude das associações entre o S/O (desfecho) e os diferentes fatores familiares analisados. Foram construídos dois modelos para a regressão de Poisson, um univariado e um ajustado pelas variáveis de confusão (atividade física e hábitos alimentares), no qual foram inseridas as variáveis de confusão que apresentaram valores de associação até $20 \%$ (ambas) no modelo univariado (método stepwise). Valores de significância $(p)$ inferiores a $5 \%$ foram considerados significativos e o tratamento estatístico foi efetuado no software STATA 8.0

\section{Resultados}

A Tabela 2 apresenta as informações gerais da amos- tra, divididas por âmbito escolar. Na comparação estabelecida entre os estudantes de ambas as redes de ensino, não foram observadas diferenças significativas do ponto de vista estatístico para as variáveis: idade cronológica, estatura, prática habitual de atividades físicas e ocorrência de hábitos alimentares inadequados.

Quando comparados com os estudantes da rede pública de ensino, os estudantes da rede privada apresentaram maiores valores de peso corporal, IMC e, consequentemente, maior ocorrência de excesso de peso (Pública $=19,4 \%$ versus Privada $=32,6 \%$; $p=0,001)$.

Aproximadamente $30 \%$ dos alunos da rede pública situaram-se nos estrados socioeconômicos mais baixos, enquanto esse valor foi inferior a $2 \%$ na rede privada de ensino. Nove entre dez pais dos alunos da rede privada de ensino apresentam escolaridade superior a nove anos, ao mesmo passo que, na rede pública de ensino, aproximadamente $50 \%$ dos responsáveis apresentaram a mesma escolaridade. Não houve associação significativa $(p=0,225)$ entre sexo e âmbito escolar (dados não apresentados).

As famílias dos adolescentes matriculados na rede privada de ensino apresentaram menor número de irmãos do que as famílias dos adolescentes matriculados na rede pública $(p=0,001)$. A quantidade de aparelhos televisores também apresentou uma associação positiva com a rede privada de ensino: aproximadamente um terço das residências dos adolescentes dessa rede possuem mais do que quatro desses aparelhos.

A Tabela 3 apresenta as associações entre a presença do excesso de peso (variável desfecho) e os diferentes fatores familiares envolvidos neste estudo

Tabela 2

Informações gerais dos adolescentes analisados agrupados de acordo com a rede de ensino. Presidente Prudente, São Paulo.

\begin{tabular}{lccc}
\hline Variáveis & $\begin{array}{c}\text { Rede Pública } \\
(\mathrm{N}=1309)\end{array}$ & $\begin{array}{c}\text { Rede Particular } \\
(\mathrm{N}=470)\end{array}$ \\
\hline Idade (anos; Mediana [DI]) & $13,6(2,8)$ & $14,1(3,1)$ & $0,557^{*}$ \\
Massa corporal (kg; Mediana [DI]) & $50,9(16,4)$ & $54,5(20,8)$ & $0,001^{*}$ \\
Estatura (m; Mediana [DI]) & $1,60(0,14)$ & $1,61(0,16)$ & $0,800^{*}$ \\
IMC (kg/m2; Mediana [DI]) & $19,4(4,6)$ & $20,9(5,6)$ & $0,001^{*}$ \\
Esportes (sim; [\%]) & 15,7 & 12,6 & 0,125 \\
Alimentação inadequada. (sim; [\%]) & 5,4 & 3,6 & 0,181 \\
Sobrepeso/Obesidade (\%) & 19,4 & 32,6 & 0,001 \\
\hline
\end{tabular}

$\mathrm{DI}=$ diferença entre Q3 e Q1; *teste U de Mann-Whitney; IMC= índice de massa corporal. 
(variáveis independentes). $\mathrm{Na}$ rede pública de ensino, o sexo masculino $(\mathrm{RP}=1,32[1,04-1,66]) \mathrm{e}$ uma maior escolaridade materna $(\mathrm{RP}=1,42[1,12$ $1,79]$ ) associaram-se com o excesso de peso dos adolescentes, mesmo após o ajuste dessa associação pelas variáveis de confusão (atividade física e hábitos alimentares). O menor número de irmãos também se associou ao excesso de peso $(\mathrm{RP}=1,25$ [1,01-1,53]), entretanto, após o ajuste dessa associ- ação pelas variáveis de confusão $(\mathrm{RP}=1,23[0,99$ $1,55]$ ), foram observados valores marginais de significância para a mesma $(p=0,052)$.

$\mathrm{Na}$ rede privada de ensino, a maior escolaridade paterna $(\mathrm{RP}=0,68[0,48-0,96]) \mathrm{e}$, assim como observado na rede pública, o sexo $(\mathrm{RP}=1,38[1,03-1,84])$ mantiveram-se associados ao excesso de peso mesmo após o ajuste pelas variáveis de confusão.

Tabela 3

Razão de prevalência para as associações entre os fatores familiares e a presença do excesso de peso em adolescentes de ambas as redes de ensino. Presidente Prudente, São Paulo. (N=1779).

\begin{tabular}{|c|c|c|c|c|c|c|c|c|}
\hline \multirow{3}{*}{ Fatores familiares } & \multicolumn{4}{|c|}{ Rede Pública } & \multicolumn{4}{|c|}{ Rede Particular } \\
\hline & \multicolumn{2}{|c|}{ Bruta } & \multicolumn{2}{|c|}{ Ajustada* } & \multicolumn{2}{|c|}{ Bruta } & \multicolumn{2}{|c|}{ Ajustada* } \\
\hline & $\mathrm{RP}$ & IC95\% & $\mathrm{RP}$ & IC95\% & $\mathrm{RP}$ & IC95\% & RP & IC95\% \\
\hline \multicolumn{9}{|l|}{ Condição econômica } \\
\hline Alta & 1,21 & $0,9-1,5$ & - & - & 2,97 & $0,4-18,9$ & - & - \\
\hline \multicolumn{9}{|l|}{ Sexo } \\
\hline Masculino & 1,33 & $1,0-1,6$ & 1,32 & $1,0-1,6$ & 1,35 & $1,0-1,7$ & 1,38 & $1,0-1,8$ \\
\hline \multicolumn{9}{|l|}{ Irmãos } \\
\hline$\leq 1$ irmão & 1,25 & $1,0-1,5$ & 1,23 & $0,9-1,5$ & 0,92 & $0,7-1,2$ & - & - \\
\hline \multicolumn{9}{|l|}{ Televisores } \\
\hline$\geq 4$ & 1,24 & $0,8-1,7$ & - & - & 1,14 & $0,8-1,5$ & - & - \\
\hline \multicolumn{9}{|l|}{ Escolaridade Pai } \\
\hline$\geq 9$ anos & 1,09 & $0,8-1,3$ & - & - & 0,74 & $0,5-0,9$ & 0,68 & $0,5-0,9$ \\
\hline \multicolumn{9}{|l|}{ Escolaridade Mãe } \\
\hline$\geq 9$ anos & 1,42 & $1,1-1,8$ & 1,42 & $1,1-1,8$ & 0,83 & $0,5-1,2$ & - & - \\
\hline
\end{tabular}

*ajustada por atividade física e hábitos alimentares; RP=Razão de Prevalência.

\section{Discussão}

No presente estudo, a hipótese inicial fazia referência à não existência de associação entre a ocorrência do excesso de peso e o âmbito escolar. E a segunda hipótese indicava que as associações observadas entre a presença do excesso de peso e os diferentes fatores familiares analisados comportar-seiam de maneira similar, tanto em magnitude quanto em direção, entre os adolescentes da rede privada e pública de ensino.

Nesse sentido, quando analisada separadamente, a ocorrência de excesso de peso foi próxima de $20 \%$ entre os adolescentes da rede pública e superior a $30 \%$ entre os adolescentes da rede privada de ensino. Essa maior prevalência de $\mathrm{S} / \mathrm{O}$ entre os alunos da rede privada de ensino concorda com observações efetuadas entre crianças das cidades de Feira de
Santana-BA, ${ }^{11}$ Santos-SP, ${ }^{9}$ e adolescentes das cidades de Fortaleza-CE ${ }^{8}$ e Campina Grande-PB7; rejeitando a primeira hipótese do estudo.

$\mathrm{O}$ sexo masculino esteve associado à presença do excesso de peso em ambos os âmbitos escolares e manteve-se assim, mesmo após o ajuste da associação pelas variáveis de confusão. Estudos anteriores 14,19 envolvendo adolescentes da região também apresentaram dados semelhantes, onde a ocorrência do $\mathrm{S} / \mathrm{O}$ foi superior entre os indivíduos do sexo masculino. A influência do sexo no processo de desenvolvimento do excesso de peso apresenta extrema complexidade, pois é contaminada pela ação de inúmeras variáveis de confusão como é o caso da condição socioeconômicas. ${ }^{1}$ Além disso, outros fatores associados ao desenvolvimento do excesso de peso, como é o caso da atividade física, também são fortemente influenciados por fatores sociocul- 
turais (apoio familiar). ${ }^{20}$ Sendo assim, em virtude de seu delineamento transversal, foge do alcance desse estudo examinar mais profundamente as influências do sexo nas associações em questão, mas cabe indicar sua forte participação no fenômeno analisado e a necessidade de mais pesquisas investigando seu papel em tal processo.

Marins et al.,21 analisando crianças e adolescentes de famílias de baixa renda, indicaram que um menor número de pessoas na residência associou-se positivamente ao excesso de peso. A associação observada entre excesso de peso e um número reduzido de irmãos (indicador de tamanho familiar), no presente estudo, pode indicar também uma influência desse menor número de pessoas vivendo na residência. Os possíveis mecanismos relacionados a esse fenômeno estariam atrelados a uma maior disponibilidade de alimentos e bens de consumo que se concentrariam sobre um menor número de crianças/adolescentes no núcleo familiar. Além disso, embora não especificada a forma de ação, uma maior quantidade de irmãos parece influenciar de maneira positiva um maior engajamento em atividades físicas. 22 Nesse sentido, em virtude de sua relevância, essa influência deveria ser averiguada com maior atenção em futuros experimentos.

Estudos transversais associando a presença do excesso de peso com a escolaridade dos pais são encontrados na literatura.23,24 De fato, essa associação foi detectada em ambas redes de ensino, porém, com direções de efeito diferenciadas. Entre os adolescentes da rede pública de ensino, a maior escolaridade materna associou-se positivamente com a presença do excesso de peso e, assim como observado em outras pesquisas, 6,21 caracteriza o importante papel da mãe no processo de desenvolvimento/proteção ao desenvolvimento do excesso de peso/gordura abdominal entre os filhos.

Entre os adolescentes da rede privada de ensino, a maior escolaridade paterna associou-se negativamente com o excesso de peso. Tal associação negativa pode representar a influência benéfica que um maior acesso a informações relacionadas aos malefícios gerados pelo $\mathrm{S} / \mathrm{O}$ pode proporcionar sobre seu combate. Entretanto, a existência de tal efeito protetor não tem recebido suporte por parte da lite- ratura, uma vez que detectamos apenas um estudo 5 que observou tal associação negativa entre excesso de peso e escolaridade dos pais (mães com nível superior completo) e, além disso, o valor da significância observada foi marginal $(p=0,093)$.

Nesse sentido, com base nos dados acima apresentados, rejeita-se a segunda hipótese testada, uma vez que, dependendo da rede de ensino analisada, com exceção do sexo, todos os demais fatores familiares associaram-se com o excesso de peso de maneiras distintas. Este comportamento distinto observado nas associações entre excesso de peso e seus fatores de risco dependendo do ambiente analisado, também foram observados por Fernandes et al., 5 entre adolescentes de alta e baixa condição econômica, e indicam que o $\mathrm{S} / \mathrm{O}$ adquire características próprias dependendo do ambiente onde é analisado.

A principal limitação do presente estudo reside em seu delineamento transversal, característica essa que possibilita a análise de associações, mas impede o estabelecimento de relações de causalidade entre as variáveis analisas. Nesse sentido, as inferências das associações aqui observadas devem ser efetuadas com cautela e futuros estudos de tendência temporal devem ser realizados não somente com esta amostra, mas também em outras regiões do país. Além disso, dada a relevância do assunto, informações referentes à questão maturacional deveriam ser inclusas em futuros trabalhos.

Dessa forma, conclui-se que, com exceção do sexo, o excesso de peso associou-se com diferentes fatores familiares nas escolas da rede pública e privada. Além disso, foi observado que, dependendo da rede de ensino onde é investigado, o excesso de peso associou-se de diferentes maneiras com o mesmo fator familiar, caso da escolaridade paterna/materna.

\section{Agradecimentos}

Os autores agradecem à Coordenação de Aperfeiçoamento do Pessoal de Nível Superior (CAPES) pela bolsa de Mestrado cedida ao estudante Rômulo Araújo Fernandes durante o segundo semestre do ano de 2007.

\section{Referências}

1. Monteiro CA, Conde WL, Popkin BM. Income-specific trends in obesity in Brazil: 1975-2003. Am J Public Health. 2007; 97: 1808-12.
2. Wang Y, Monteiro C, Popkin BM. Trends of obesity and underweight in older children and adolescents in the United States Brazil China and Russia. Am J Clin Nutr. 2002; 75: 971-7. 
3. Ferreira AP, Oliveira CE, França NM. Metabolic syndrome and risk factors for cardiovascular disease in obese children: the relationship with insulin resistance (HOMA-IR). J Pediatr (Rio J). 2007; 83: 21-6.

4. Moreira SR, Ferreira AP, Lima RM, Arsa G, Campbell CS, Simões HG, Pitanga FJG, França NM. Predicting insulin resistance in children: anthropometric and metabolic indicators. J Pediatr (Rio J). 2008; 84: 47-52.

5. Fernandes RA, Casonato J, Christofaro DG, Ronque ER, de Oliveira AR, Freitas Júnior IF. Riscos para o excesso de peso entre adolescentes de diferentes classes socioeconômicas. Rev Assoc Med Bras. 2008; 54: 334-8.

6. Ortega FB, Ruiz JR, Sjöström M. Physical activity, overweight and central adiposity in Swedish children and adolescents: the European Youth Heart Study. Int J Behav Nutr Phys Act. 2007; 4: 61.

7. Araújo Nunes MM, Figueiroa JN, Alves JG. Overweight, physical activity and foods habits in adolescents from different economic levels, Campina Grande (PB). Rev Assoc Med Bras. 2007; 53: 130-4.

8. Campos LA, Leite AJ, Almeida PC. Prevalência de sobrepeso e obesidade em adolescentes escolares do município de Fortaleza, Brasil. Rev Bras Saúde Matern Infant. 2007; 7: 183-90.

9. Costa RF, Cintra IS, Fisberg M. Prevalência de sobrepeso e obesidade em escolares da cidade de Santos SP. Arq Bras Endocrinol Metab. 2006; 50: 60-7.

10. Brasil LMP, Fisberg M, Maranhão HS. Excesso de peso em escolares em região do Nordeste Brasileiro: contraste entre as redes de ensino pública e privada. Rev Bras Saúde Matern Infant. 2007; 7: 405-12.

11. Oliveira AM, Oliveira AC, Almeida MS, Oliveira N, Adan L. Influence of the family nucleus on obesity in children from northeastern Brazil: a cross-sectional study. BMC Public Health. 2007; 7: 235-45.

12. Scherr C, Magalhães CK, Malheiros W. Lipid profile analysis in school children. Arq Bras Cardiol. 2007; 89: 6570.

13. Veugelers PJ, Fitzgerald AL. Effectiveness of school programs in preventing childhood obesity: a multilevel comparison. Am J Public Health. 2005; 95: 432-5.
14. Fernandes RA, Kawaguti SS, Agostini L, Oliveira AR, Ronque ERV, Freitas Júnior IF. Prevalência de sobrepeso e obesidade em alunos de escolas privadas do município de Presidente Prudente - SP. Rev Bras Cineantropom Desempenho Hum. 2007; 9: 21-7.

15. Gordon CC, Chumlea WC, Roche AF. Stature, recumbent length and weight. In: Lohman TG, Roche AF, Martorel R, editors. Anthropometric standardization reference manual. Champaign: Human Kinetics Books; 1988. p. 3-8.

16. Cole TJ, Bellizzi MC, Flegal KM, Dietz WH. Establishing a standard definition for child overweight and obesity worldwide: international survey. BMJ. 2000; 320: 1240-3.

17. Baecke JAH, Burema J, Frijters JE. A short questionnaire for the measurement of habitual physical activity in epidemilogical studies. Am J Clin Nutr. 1982; 36: 936-42.

18. IBOPE (Instituto Brasileiro de Opinião Pública e Estatística) [Internet]. Levantamento socioeconômico2000-IBOPE. Rio de Janeiro. [acesso em 2 fev 2003]. Disponível em: http://www.anep.org.br

19. Fernandes RA, Rosa CS, Buonani C, Oliveira AR, Freitas Júnior IF. The use of bioelectrical impedance to detect excess visceral and subcutaneous fat. J Pediatr (Rio J). 2007; 83: 529-34.

20. Gonçalves H, Hallal PC, Amorim TC, Araújo CL, Menezes AM: Sociocultural factors and physical activity level in early adolescence. Rev Panam Salud Publica. 2007; 22: 246-53.

21. Marins VM, Almeida RM, Pereira RA, Azevedo Barros MB. The relationship between parental nutritional status and overweight children/adolescents in Rio de Janeiro, Brazil. Public Health. 2004; 118: 43-9.

22. Hallal PC, Wells JC, Reichert FF, Anselmi L, Victora CG: Early determinants of physical activity in adolescence: prospective birth cohort study. BMJ. 2006; 332: 1002-7.

23. Suñé FR, Dias-da-Costa JS, Olinto MT, Pattussi MP. Prevalência e fatores associados para sobrepeso e obesidade em adolescentes de uma cidade no Sul do Brasil. Cad Saúde Pública. 2007; 23: 1361-71.

24. Terres NG, Pinheiro RT, Horta BL, Pinheiros KA, Hotra LL. Prevalence of factors associated to overweight and obesity in adolescents. Rev Saúde Pública. 2006; 40: 1-6.

Recebido em 30 de maio de 2008

Versão final apresentada em 17 de agosto de 2009

Aprovado em 18 de setembro de 2009 